Erratum

Nervenarzt 2019.90:1292

https://doi.org/10.1007/s00115-019-0731-5

Online publiziert: 17. Mai 2019

(c) Springer Medizin Verlag $\mathrm{GmbH}$, ein Teil von Springer Nature 2019

Jacqueline M. Kondziela' - Juliane Schulz ${ }^{1}$ Bernhard Brunst ${ }^{2}$. Simone Fuchs ${ }^{3}$. Stefan Gerlinger ${ }^{4}$ Birgit Neif ${ }^{5}$. Henrike Staab-Kupke ${ }^{3}$. Silke Vasileiadis ${ }^{1}$. Peter Brodisch ${ }^{6}$. Susanne Knake ${ }^{1}$. Tobias Kniess ${ }^{7}$. Bernd Schade ${ }^{5}$. Bernd A. Neubauer ${ }^{4} \cdot$ Felix Rosenow $^{8} \cdot$ Susanne Schubert-Bast ${ }^{8,9}$. Adam Strzelczyk ${ }^{1,8} \cdot$ Laurent M. Willems ${ }^{8}$

'Epilepsiezentrum Hessen und Klinik für Neurologie, Philipps-Universität Marburg, Marburg, Deutschland ${ }^{2}$ Epilepsieberatung Diakonisches Werk Hochtaunus, Frankfurt am Main, Deutschland ${ }^{3}$ Epilepsieberatung Unterfranken, Stiftung Juliusspital, Würzburg, Deutschland

${ }^{4}$ Abteilung für Kinderneurologie und Sozialpädiatrie, Justus-Liebig-Universität Gießen, Gießen, Deutschland

${ }^{5}$ Klinik für Neurologie, Hephata-Klinik, Schwalmstadt-Treysa, Deutschland

${ }^{6}$ Epilepsieberatung, Innere Mission München, Diakonie in München und Oberbayern e. V., München, Deutschland

${ }^{7}$ Klinik für Neurologie, Campus Rhön Klinikum AG, Bad Neustadt a.d. Saale, Deutschland

${ }^{8}$ Epilepsiezentrum Frankfurt Rhein-Main, Zentrum der Neurologie und Neurochirurgie, GoetheUniversität Frankfurt, Frankfurt am Main, Deutschland

${ }^{9}$ Schwerpunkt Neurologie, Neurometabolik, und Prävention der Klinik für Kinder- und Jugendmedizin, Goethe-Universität Frankfurt, Frankfurt am Main, Deutschland

\title{
Erratum zu: Akzeptanz, Bedarf, Konsultationsgründe und Beratungsoutcome von Epilepsieberatung in Hessen und Unterfranken
}

\section{Erratum zu:}

Nervenarzt 2019

https://doi.org/10.1007/s00115-0190676-8

Die ursprünglich erschienene Onlineversion des Beitrags enthielt eine falsche Prozentangabe $\mathrm{zu}$ den betroffenen erwachsenen Patienten im Hinblick auf eine Weiterbeschäftigung. Diese Prozentangabe wurde von den Autoren nachträglich korrigiert.

Im Abschnitt Outcome der Epilepsieberatung im Hinblick auf Arbeitsplatzund Schulplatzerhalt muss daher der letzte Satz lauten:

Bei 34,0\% $(n=18)$ der betroffenen erwachsenen Patienten konnte keine Weiterbeschäftigung erreicht werden, bei $17,2 \%(n=5)$ der Kinder und Jugendlichen war trotz Beratung ein Wechsel der Schulform hin zu einer Institution mit niedrigerem Zielabschluss nötig.

Ebenso muss diese Angabe in Tab. 2 in der letzten Zeile, mittlere Spalte $34 \%$ lauten, statt wie ursprünglich angegeben $4 \%$.

Wir bitten dies zu beachten.

\section{Korrespondenzadresse}

Prof. Dr. Adam Strzelczyk, MHBA

Epilepsiezentrum Frankfurt Rhein-Main, Zentrum der Neurologie und Neurochirurgie, Goethe-Universität Frankfurt

Schleusenweg 2-16, 60528 Frankfurt am Main, Deutschland

strzelczyk@med.uni-frankfurt.de

Die Online-Version des Originalartikels ist unter https://doi.org/10.1007/s00115-019-0676-8zu finden. 